\title{
Multi-scale Modeling of Dynamics and Ignition to Flame Transitions of High Pressure Stratified n-heptane/toluene Mixtures
}

\author{
Weiqi Sun ${ }^{1}$, Sang Hee Won ${ }^{1}$, Xiaolong Gou ${ }^{2}$, Yiguang Ju ${ }^{1}$ \\ ${ }^{1}$ Department of Mechanical and Aerospace Engineering \\ Princeton University, New Jersey 08544, USA \\ ${ }^{2}$ College of Power Engineering \\ Chongqing University, Chongqing 400030, PR China
}

\section{Corresponding Author}

Prof. Yiguang Ju

Department of Mechanical and Aerospace Engineering

Princeton University

Princeton, NJ 08544, USA

Phone: +1 6092585644

Email: yju@Princeton.EDU

\section{Colloquium}

Laminar Flames

\section{Total length of paper: 5853 (6200 limit)}

Main text: 3557

Figures with caption: 1545

Equations: 122

References: 629

Keywords: Fuel stratification; Ignition to flame transition; Low temperature chemistry; Engine knocking; n-alkanes and aromatics 


\title{
Multi-scale Modeling of Dynamics and Ignition to Flame Transitions of High Pressure Stratified n-heptane/toluene Mixtures
}

\author{
Weiqi Sun ${ }^{1}$, Sang Hee Won ${ }^{1}$, Xiaolong Gou ${ }^{2}$, Yiguang Ju ${ }^{1}$ \\ ${ }^{1}$ Department of Mechanical and Aerospace Engineering \\ Princeton University, New Jersey 08544, USA \\ ${ }^{2}$ College of Power Engineering \\ Chongqing University, Chongqing 400030, PR China
}

\begin{abstract}
The transitions from ignition to flames as well as the combustion dynamics in stratified nheptane and toluene mixtures are numerically modeled by a correlated dynamic adaptive chemistry method coupled with a multi-timescale method (CO-DAC/MTS) in a onedimensional constant volume chamber. The study attempts to answer how the kinetic difference between n-alkanes and aromatics leads to different ignition to flame transitions and knocking-like acoustic wave formation at low temperature and engine pressure conditions with fuel stratification. It is found that the low temperature chemistry (LTC) and fuel stratification of $n$-heptane leads to the formation of multiple ignition fronts. Four different combustion wave fronts, a low temperature ignition (LTI) front followed by a high temperature ignition (HTI) front, a premixed flame (PF) front, and a diffusion flame (DF) front, are demonstrated. The fast LTI and HTI wave front propagation leads to a shock-like strong acoustic wave propagations, thus strongly modifying the dynamics of the subsequent diffusion and premixed flame fronts. On the other hand, for the toluene mixture, due to the lack of LTC, only two combustion wave fronts are formed, a HTI front and a premixed flame front, exhibiting stable flow field and no formation of shocklike acoustic wave. The dynamics of transition from combustion to shock waves is further analyzed by using a modified Burgers' equation. The analysis for n-heptane/air mixture indicates that both the onset of LTI and the strong dependency of HTI on the equivalence ratio can either promote or attenuate the transition from strong acoustic wave to shock wave. However, the toluene/air mixture exhibits no coupling with acoustic wave, suggesting that the rich LTC reactivity with fuel stratification, specific to the n-alkane chemistry, can lead to knocking and acoustic formation.
\end{abstract}




\section{Introduction}

Due to the energy sustainability and global warming issues, increasing the fuel efficiency of internal combustion engines and reducing their emissions are of global importance. Recently, there is increasing interest in developing more efficient and lower emission internal combustion engines, operating at the higher compression ratio and ultra-lean conditions. These advanced engines include the homogeneous charge compression ignition (HCCI) engine [1], the reactivity controlled compression ignition (RCCI) engine [2], and the next generation twin annular premixing swirler (TAPS) engine [3,4]. Unfortunately, there are still several technical challenges for HCCI and RCCI engines due to the increased ignition sensitivity to fuel chemistry at low and intermediate temperature ranges. Stochastic engine knocking at high engine load and at high compression ratio is one of the major challenges. The control of ignition timing and heat release rate via fuel stratification or fuel additives (adding aromatics or cetane number enhancers) has been found to be an effective way for controlling engine knocking at different engine loads. [5,6,7]. However, the understanding of combustion processes and fundamental mechanism of ignition to knocking transition for stratified fuel mixtures with both n-alkanes and aromatics engine conditions is still limited due to the complexity of low temperature chemistry (LTC) with negative temperature coefficient (NTC) regime and combustion properties of large hydrocarbon fuels [8], as well as the complicated multiple flame regimes [9].

Several numerical modeling studies for HCCI engines have been carried out to understand the details of ignition to flame transition. Unfortunately, due to limitation of computation power, most of studies have focused on small hydrocarbons $[10,11]$. Recently, a multi-scale modeling of n-heptane/air mixture in a one-dimensional spark assisted HCCI engine was conducted, identifying four different flame regimes [9]. More recently, auto-ignition of DME/air mixtures with temperature and fuel stratifications accounting for the exhaust gas recirculation (EGR) was modeled by using direct numerical simulations $[12,13,14]$, but under the short computation times as well as the limited small computation domain. Consequently, it was not able to capture the transition from ignition to flames. Therefore, it can be regarded that the combustion phenomena from ignition to flame transitions at NTC conditions for stratified large hydrocarbons have not been investigated. Considering the strong chemical kinetic couplings between alkanes and aromatics [15], which is common in real fuels [16,17], it is also of interest to investigate how the ignition to flame transitions occur differently in a stratified flow between alkanes and aromatics. More importantly, the relation between engine knocking and ignition to flame transitions at NTC conditions is not well-understood either.

The goal of this study is to answer following three questions: 1) How does auto-ignition transit flames where there is a fuel stratification at NTC condition? 2) How does ignition to flame transition affect combustion oscillation and knocking-like acoustic wave 
formation? 3) How does the kinetic difference between n-alkanes (with NTC) and aromatics (no NTC) affect ignition to flame transitions and flame dynamics? Firstly, the high pressure, unsteady ignition to flame transition and combustion dynamics in onedimensional stratified n-heptane and toluene mixtures are modeled using a correlated dynamic adaptive (CO-DAC) method [18] coupled with a hybrid multi-timescale (HMTS) method [19]. The formations of different ignition and flame regimes are examined and compared. The dynamics of the formation of flame oscillation and strong pressure waves are analyzed by using a simplified Burgers' equation.

\section{Numerical Models}

In order to simplify the complicated ignition to flame transition and combustion dynamics of stratified alkane/aromatics-air mixtures in a large domain at engine-like conditions without the huge computational cost, we model a stratified mixture with a given equivalence ratio $(\varphi)$ gradient in a one-dimensional constant volume domain.

The distribution of equivalence ratio is to mimic a simplified fuel stratification caused by the pulsed fuel injections in engines. Although real engines are turbulent, the laminar assumption can still provide insights of the fundamental phenomena. The length of the domain is $5 \mathrm{~cm}$. In order to understand the kinetic effect the low temperature chemistry on ignition to flame transition, two fuel molecules are chosen; n-heptane exhibits rich low temperature reactivity with NTC behavior, whereas toluene follows the simple Arrhenius-type reactivity without NTC behavior. A comprehensively reduced chemical kinetic model (170 species and 962 reactions) for n-heptane/toluene mixture produced with the multi-generation path flux analysis method [20,21] from the detailed model is used in this study [22]. Details of model performance of ignition delay prediction can be found in the supplementary material. Unless otherwise stated, the initial pressure and temperature is $40 \mathrm{~atm}$ and $800 \mathrm{~K}$, respectively. The reflective and zero gradient boundary conditions are applied, respectively, for velocity and all other scalar variables.

To utilize large chemical kinetic model efficiently in the computations, a correlated dynamic adaptive chemistry (CO-DAC) method [18] coupled with a hybrid multitimescale (HMTS) method [19] are developed and integrated to solve the chemical reactions. The CO-DAC method reduces the size of kinetic model on the fly by using path flux analysis (PFA) method [20,21] and using the correlations of local reactivity in phase space [18] over both time and space domains. The multi-level local grid refinement is conducted by using adaptive simulation of unsteady reactive flow (ASURF) code $[23,24]$. In ASURF, adaptive and unstructured grids are generated at the beginning of every computation steps based on the local length scales, i.e. the local pressure gradient, density gradient and velocity gradient. Based on the adaptive grids, the splitting fractional-step procedure [25] is applied to decouple the chemical reaction terms and transport terms. The chemical reaction terms, which are described by a first order ODE 
system, are solved in the first fractional step. Then the thermodynamic state variables after solving chemical reaction terms are used as the initial conditions in the second fractional step to compute the transport terms, which include unsteady, convection and diffusion terms and described by a second order PDE system. Once the transport terms are solved, all the variables can be updated to next time step. The ASURF code has been validated extensively in our previous studies and collaborations with other researchers $[26,27]$. In the modeling of this paper, the smallest grid size after mesh adaption is $16 \mu \mathrm{m}$. To further improve the spatial resolution, a third-order weighted essentially nonoscillatory (WENO) scheme is applied to solve the PDE system in the second fractional step based on the adaptive and unstructured grids [28,29,30,31,32]. With the above CODAC and higher-order WENO scheme, the original ASURF is updated to ASURF+. As such, the unsteady, reactive flow with large kinetic mechanism is solved adaptively by using ASURF+.

\section{Results and Discussion}

\subsection{Dynamics and combustion regimes in stratified n-heptane/air mixture}

Prior to the detailed computations, in order to envision the dependency of low and high temperature ignitions on the equivalence ratio, the ignition delay times of homogenous nheptane/air and toluene/air mixtures at different equivalence ratios are calculated firstly at the initial condition of $40 \mathrm{~atm}$ and $800 \mathrm{~K}$. The results are summarized as the blue and red curves in Fig. 1. In case of n-heptane/air mixtures, two distinctive ignition processes are observed, known as two-stage ignition. The first-stage ignition is controlled by the radical pool initiation predominantly by the low temperature chemistry, so called as low temperature ignition (LTI). After the onset of LTI, the $\mathrm{HO}_{2}$ radical plays important role in the fuel destruction, while accumulating the $\mathrm{H}_{2} \mathrm{O}_{2}$ with the gradual increase of temperature. When the mixture temperature reaches the critical condition to initiate the thermal decomposition of $\mathrm{H}_{2} \mathrm{O}_{2}$ to form two active $\mathrm{OH}$ radicals, then the second-stage ignition occurs, known as high temperature ignition (HTI) [8]. The calculated results for n-heptane/air mixtures show that the LTI is weakly dependent on the equivalence ratio, whereas the HTI exhibits the strong sensitivity to the fuel equivalence ratio. Particularly, with the increase of equivalence ratio, the time interval between LTI and HTI decreases dramatically. The faster transition from LTI to HTI at the richer equivalence ratio indicates that the fuel stratification might induce the unexpected chemistry/transport coupling or interaction during the ignition to the flame transition processes. Compared to the n-heptane/air mixture, the toluene/air mixture shows relatively simpler ignition behaviors, demonstrating only the HTI process for all computed equivalence ratios. The HTI delay times of toluene/air mixture is about two orders of magnitude slower than those of n-heptane/air mixture. 
The computations in this study aim to simulate the circumstance of multiple fuel injection strategy, where the small amount of fuel is pre-injected into the chamber to form lean homogeneous fuel/air mixture prior to the main fuel injection. Thus, initial fuel distribution in the chamber is formulated as below for both $\mathrm{n}$-heptane and toluene cases.

$$
\Phi=\left\{\begin{array}{c}
5.9 *(3-x)+0.3, x<3 \mathrm{~cm} \\
0.3, x \geq 3 \mathrm{~cm}
\end{array}\right.
$$

The distribution of fuel is depicted in the insert of Fig. 1. The right half domain with constant equivalence ratio as 0.3 represents the typical HCCI engine working condition $[12,13]$. In order to incorporate the fuel injection and subsequent fuel/air mixing process, a large gradient of equivalence ratio is adopted as an initial condition on the left half domain. Consequently, the initial boundary condition on the left is set to the equivalence ratio of 18 .

Figure 2 shows the trajectories of the locations of local maximum heat release rates of the stratified n-heptane/air mixture in a function of time ( $t$ ) with the initial condition at 40 atm and $800 \mathrm{~K}$. In order to differentiate ignition wave fronts and flame fronts observed in this computation, the reaction terms and diffusion terms in governing equations have been traced along the trajectories of maximum heat release rates. When the reaction terms dominate over diffusion terms, the wave front is defined to be controlled by ignition characteristics (ignition wave front). On the other hand, it is defined as flame front when the diffusion term becomes comparable to the reaction term, as a typical configuration of flame structure.

Although the LTI delay time is a weak function of equivalence ratio, it still has the shortest delay time at the fuel rich condition. Thus, the LTI occurs at the fuel richest location $(\mathrm{x}=0 \mathrm{~cm})$ at $\mathrm{t}=0.62 \mathrm{~ms}$. After achieving the LTI at the fuel rich side, the LTI wave front propagates quickly to the direction of lower equivalence ratio, indicated by the steep gradient right after the onset of LTI. The estimated propagation speed of the LTI wave front is about $1400 \mathrm{~m} / \mathrm{s}$, indicating that the propagation of LTI wave front can be regarded as sequential event of LTI controlled by the weak dependency of LTI on the fuel stratification, shown in Fig. 1. Consequently, this fast propagation of LTI wave will lead to the formation of a strong pressure wave (shock). This LTI wave behavior is similar to the concept of "spontaneous" wave introduced by Zeldovich [33]. Both this LTI wave and the "spontaneous" wave exhibit the characteristics of strong dependence of propagation rate on initial conditions, as well as its independence of heat conductivity and the sound speed of the gas. At $\mathrm{t}=0.66 \mathrm{~ms}$, a new HTI ignition occurs at $\mathrm{x}=2 \mathrm{~cm}$. This HTI wave front propagates at the speed of $350 \mathrm{~m} / \mathrm{s}$ to the same direction of LTI wave front. At $\mathrm{x}=3 \mathrm{~cm}$, the LTI wave front disappears because of failing to initiate LTI at the excessively lean condition $(\varphi=0.3)$ prior to achieving HTI. HTI wave front continues to propagate toward the fuel lean side. The large pressure rise due to the large 
heat release after the HTI wave front results in a strong oscillation of the ignition front at $\mathrm{t}=0.72 \mathrm{~ms}$.

At $\mathrm{t}=0.80 \mathrm{~ms}$, it is interesting to note that the HTI wave front splits into a premixed flame front and a diffusion flame front, determined by the balance between reaction and diffusion terms in governing equations. The premixed flame front propagates into the region with uniform equivalence ratio (insert of Fig.1) at an average speed of $30 \mathrm{~m} / \mathrm{s}$ while continues to oscillate. However, the average advection speed of diffusion flame front speed is very low, stagnantly stabilized at $\mathrm{x}=3.2 \mathrm{~cm}$, but the oscillation continues with a constant frequency about $8300 \mathrm{~Hz}$ even after the disappearance of the premixed flame front. This oscillation is caused by acoustic wave reflection on the chamber wall. Similar pressure oscillations have been observed in engines when a knock occurred [34]. The result in Fig.2 demonstrates that there exist four different combustion regimes during the ignition to flame transition for n-heptane/air mixture, which has high cetane number (low octane number) [35], a low temperature ignition (LTI) regime, a high temperature ignition (HTI) regime, a premixed flame (PF) regime, and a diffusion flame (DF) regime. In addition, the fast propagation speed of HTI wave front results in strong oscillations of the subsequent diffusion and premixed flame fronts.

To understand the dynamics and structure of different wave fronts, the temperature and heat release rates at three sampling times at the LTI, HTI and PF and DF regimes marked on Fig.2 are further analyzed in a spatial coordinate. Figure 3 shows the spatial distributions of temperature (a) and the heat release rate (b) at three sampling times, respectively. It is seen that at $\mathrm{t}=0.64 \mathrm{~ms}$ (sample 1 in Fig. 2), the LTI wave front is located at $\mathrm{x}=2.3 \mathrm{~cm}$ (peak heat release) and temperature rises modestly after the LTI. At $\mathrm{t}=0.78 \mathrm{~ms}$ (sample 2 in Fig. 2), a very sharp heat release wave is located at $\mathrm{x}=3.4 \mathrm{~cm}$, leading to a large temperature rise $(2700 \mathrm{~K})$. At $0.99 \mathrm{~ms}$ (sample 3 in Fig. 2), two heat release zones are observed, the left is a diffusion flame reaction zone and the right is a premixed flame front. The premixed flame leads a lower temperature rise in the fuel lean zone $(\varphi=0.3)$ but the diffusion flame further increases the flame temperature at the interface of the fuel lean and fuel rich mixture (Fig.1). The wave front structures in Fig.3 confirm the observation of different ignition and flame regimes in Fig.2.

\subsection{Dynamics and combustion regimes in stratified toluene/air mixture}

Figure 4(a) shows the trajectories of the locations of maximum heat release rates in the toluene/air mixture initiated at $40 \mathrm{~atm}$ and $1000 \mathrm{~K}$. Since the toluene oxidation chemistry does not exhibit the LTI behavior, there are only two combustion regimes existing in the mixture evolution histories, a HTI regime and a premixed flame regime.

The onset of the HTI in toluene/air mixture occurs at $\mathrm{t}=3.3 \mathrm{~ms}$ and $\mathrm{x}=0$. After the onset of the HTI, the HTI wave front begins to propagate toward the fuel lean side. Note that 
the speed of the HTI wave front in toluene/air mixture is significantly lower $(\sim 10 \mathrm{~m} / \mathrm{s})$ than that observed in n-heptane/air mixture. The slow ignition transition of toluene/air mixture during the HTI wave propagation is clearly demonstrated in the top plot of Fig. 4(b) by plotting the temperature time history along the trajectory of maximum heat release rate. In case of n-heptane/air mixture shown in the bottom plot of Fig. 4(b), the ignition transition happens immediately within $0.15 \mathrm{~ms}$ for both LTI and HTI, whereas the transition of HTI in toluene/air mixture takes much longer $(\sim 2.5 \mathrm{~ms})$ to achieve the maximum temperature $(\sim 1600 \mathrm{~K})$ at $\mathrm{t}=5.925 \mathrm{~ms}$, where the flame structure begins to form, onset of flame. This slow ignition transition of toluene/air mixture can be attributed to the slower ignition delays, as well as a strong dependence of HTI delays on the equivalence ratio as the HTI wave front propagates toward fuel lean side, depicted in Fig. 1.

Figure 5 shows the spatial profiles of temperature (a) and the heat release rate (b) at two sampling times marked in Fig.4 (a). After completing the HTI transition at $\mathrm{t}=5.925 \mathrm{~ms}$ $(\sim 1600 \mathrm{~K})$, thus achieving the onset of flame, two premixed flame fronts are formed at $\mathrm{x}$ $=2.2 \mathrm{~cm}$ and propagate in opposite directions with the propagation speed of $20 \mathrm{~m} / \mathrm{s}$ (toward fuel rich side) and $40 \mathrm{~m} / \mathrm{s}$ (toward fuel lean side), respectively. Comparing to the n-heptane case where DF and PF braches are followed after very fast LTI and HTI transitions, the slow HTI ignition transition provides extra time for flow to be further mixed. Consequently, two premixed flames propagating toward fuel rich and lean sides can be seen by plotting spatial profiles of temperature (Fig. 5(a)) and heat release rates (Fig. 5(b)). Although not shown in the figure, the completion of HTI consumes entire oxygen near $\mathrm{x}=2.2 \mathrm{~cm}$, thus prohibiting the formation of diffusion flame.

The comparison between the combustion regimes of the stratified n-heptane/air mixture and toluene/air mixture clearly demonstrated that the low temperature chemistry is the key factor of formation of the four regime behavior in n-heptane/air mixture. The weak dependence of LTI to the equivalence ratio results in a sharp, fast propagated low temperature ignition front. After the HTI, the increase of overall temperature amplifies the pressure variation across the ignition front and leads to the strong oscillation afterward.

\subsection{Dynamical Analysis of Ignition to Shock Wave Formation}

Figure 6 shows the profiles of pressure (a) and velocity (b) in the n-heptane/air mixture, respectively, at the three sampling times marked in Fig. 2. As mentioned above, in the LTI regime, the supersonic ignition front $(1400 \mathrm{~m} / \mathrm{s})$ can induce a shock wave. The flame front oscillation frequency shown in Fig. 2 is about $8300 \mathrm{~Hz}$. Figure 6(a) clearly demonstrates that at $\mathrm{t}=0.64 \mathrm{~ms}$, a sharp pressure jump is caused by the LTI ignition front. The pressure increase after the LTI is more than $20 \mathrm{~atm}$. In the HTI regime of $\mathrm{t}=0.78 \mathrm{~ms}$, the amplitude of pressure rise is increased to $40 \mathrm{~atm}$ due the larger heat release. This 
large pressure rise may lead to large velocity fluctuation as shown in Fig. 6(b) and the possible transition to knocking, occurring in a real internal combustion engine. In order to understand the effect of the fuel stratification on the shock (knocking) formation, a modified Burgers' equation [36] analysis is conducted. The Burgers' equation represents a typical development of front discontinuities (e.g. shock wave). Consider the Burger's equation coupled with pressure gradient, neglecting the viscous terms in a one dimensional flow, we can have:

$$
\frac{\partial u}{\partial t}+u \frac{\partial u}{\partial x}=-\frac{1}{\rho} \frac{\partial p}{\partial x}
$$

If we define the local velocity gradient,

$$
\frac{\partial u}{\partial x}=A
$$

And further assume the constant density, we have,

$$
\frac{D A}{D t}=-A^{2}-\frac{\partial^{2}(p / \rho)}{\partial x^{2}}
$$

If there is no pressure gradient due to the heat release in the flow, the development of $A$ only depends on $-A^{2}$, which is always negative. Thus, when A is less than zero initially, it will always develop to a shock. If temperature/pressure gradient exists due to LTI or HTI in a concentration gradient, the second term on the right hand side will modify the transition to shock wave. Figure 7 shows the influence of pressure gradient $\left(P_{1}=\right.$ $\left.200 x^{2} \mathrm{~Pa} ; P_{2}=-400 x^{2}+1600 \mathrm{~Pa}\right)$ to the shock formation in a one dimensional flow with two open ends and initiated with constant negative velocity gradient. It is found that the pressure gradient can either promote or retard the shock formation, depending on the pressure gradient and the sign of the initial velocity gradient.

In the study of the stratified n-heptane/air mixture in section 3.1, the heat release from low temperature combustion affects the pressure variation in the flow. The equivalence ratio gradient leads to the heat release rate gradient and, therefore, provides a favorable pressure gradient, which can accelerate the formation of knocking or shock-lets.

\section{Conclusions}

The ignition to flame transition and combustion dynamics of stratified n-heptane and toluene mixtures are modeled by a correlated dynamic adaptive chemistry method coupled with a multi-timescale method. The results demonstrate that in the n-heptane mixture, there are four combustion regimes, a LTI regime, a HTI regime, a premixed flame regime, and a diffusion flame regime. It is found that in the LTI regime, a supersonic ignition front coupled with a strong pressure wave is formed due to the weak dependence of LTI delay time on equivalence ratio. Increased heat release in HTI further 
increases the strength of pressure wave and led to strong oscillations in pressure, velocity, and premixed and diffusion flame fronts. On the other hand, for toluene mixture, which doesn't have low temperature chemistry, only two combustion waves, a HTI regime and a premixed flame regime, are observed. No pressure waves and flame oscillation are observed. Therefore, the differences in chemical kinetics of alkanes and aromatics have different impact on flame regimes and pressure wave formation. The present results show that alkanes with rich LTC promote knocking. The dynamical analysis of ignition to shock formation in a stratified mixture shows that fuel stratification can either accelerate or decelerate shock (knock) formation, depending on the directions of velocity gradient and the concentration gradient. The present study provides insights to understand the combustion dynamics of stratified combustion and the mechanism of knocking.

\section{Acknowledgments}

This work is supported by the Army Research Grant W911NF-12-1-0167 for multi-scale modeling. SH Won is supported by the Energy Frontier Research Center (EFRC) and the Department of Energy, Office of Basic Energy Sciences, under Award Number DeSC0001198. 


\section{Figure Captions}

Figure 1. Calculated HTI and LTI delay times for zero-dimensional homogeneous nheptane/air (blue lines) and toluene/air (red line) mixtures in a function of equivalence ratio at the initial condition of $40 \mathrm{~atm}$ and $800 \mathrm{~K}$. Insert shows the initial condition of fuel distribution used in one-dimensional simulations.

Figure 2. Trajectories of maximum heat release rate in the stratified n-heptane/air mixture at the initial condition of $40 \mathrm{~atm}$ and $800 \mathrm{~K}$.

Figure 3. Profiles of maximum temperature (a) and maximum heat release rate (b) for nheptane/air mixture at three sampling points, $\mathrm{t}=0.64,0.78$ and $0.99 \mathrm{~ms}$, marked in Fig. 2.

Figure 4. (a).Trajectories of maximum heat release rate in the stratified toluene/air mixture at the initial condition of 40 atm and $1000 \mathrm{~K}$. (b). Temperature time history along the trajectory of maximum heat release rate during ignition stages of toluene (top plot) and n-heptane (bottom plot).

Figure 5. Profiles of maximum temperature (a) and maximum heat release rate (b) for ntoluene/air mixture at two sampling points, $\mathrm{t}=5.925$ and $6.080 \mathrm{~ms}$, marked in Fig. 4.

Figure 6. Profiles of pressure (a) and velocity (b) for n-heptane/air mixture at three sampling points, $\mathrm{t}=0.64,0.78$ and $0.99 \mathrm{~ms}$, marked in Fig. 2.

Figure 7. Pressure gradient effects on the acceleration/deceleration of shock formation, analyzed with Burgers' equation. 


\section{References}

[1] F. Zhao, TW Asmus, D. N. Assanis, J. E. Dec, J. A. Eng, P. M. Najt, SAE International, 2003.

[2] D. S. Kim, C. S. Lee, Fuel, 85 (2006) 695-704.

[3] Dodds, W. J. (2005). "Twin Annular Premixing Swirler (TAPS) Combustor."The Roaring 20th Aviation Noise \& Air Quality Symposium.

[4] J.E. Dec, Proc. Combust. Inst. 32 (2009) 2727-2742.

[5] M. Sjoberg, J.E. Dec, N. Cernansky, SAE Technical Paper Series, Paper No. 200501-0113, 2005.

[6] W. Hwang, J.E. Dec, M. Sjoberg, SAE Technical Paper Series, Paper No. 2007-014130, 2007.

[7] R.D. Reitz, Directions in internal combustion engine research, Combust. Flame 160 (2013) 1-8.

[8] H.J. Curran, P. Gaffuri, W.J. Pitz, C.K. Westbrook, Combust. Flame 114 (1998) 149-177.

[9] Y. Ju, W. Sun, M.P. Burke, X. Guo and Z. Chen, Proc. Combust. Inst. 33 (2011) 1245-1251.

[10] J.H. Chen, E.R. Hawkes, R. Sankaran, S.D. Mason, H.G. Im, Combust. Flame 145 (2006) 128-144.

[11] Hawkes, R. Sankaran, P.P. Pébay, J.H. Chen, Combust. Flame 145 (2006) 145-159.

[12] H.A. El-Asrag, Y. Ju, Combustion Theory and Modelling, 17 (2013) 316-334.

[13] H.A. El-Asrag, Y. Ju, Combust. Flame 161 (2014), 256-269.

[14] Zhang, H., Hawkes, E. R., Chen, J. H., \& Kook, S. Proc. Combust. Inst. 34 (2012) 803-812.

[15] S. H. Won, W. Sun, Y. Ju, Combust. Flame 157 (2010) 411-420.

[16] S. Dooley, S. H. Won, M. Chaos, J. Heyne, Y. Ju, F. L. Dryer, K. Kumar, C.-J. Sung, H. Wang, M. A. Oehlschlaeger, R. J. Santoro, T. A. Litzinger, Combust. Flame 157 (2010) 2333-2339.

[17] S. Dooley, S. H. Won, J. Heyne, T. I. Farouk, Y. Ju, F. L. Dryer, K. Kamar, X. Hui, C.-J. Sung, H. Wang, M. A. Oehlschlaeger, V. Iyer, S. Iyer, T. A. Litzinger, R. J. Santoro, T. Malewecki, K. Brezinsky, Combust. Flame, 159 (2012) 1444-1466.

[18] W. Sun, H. A. El-Asrag, Y. Ju, "Multi-timescale Modeling of Ignition and Flame Propagation of Diesel Surrogate Fuel Mixtures," $8^{\text {th }}$ U.S. National Combustion Meeting, May 19-22, 2013, Utah, USA, Paper number 070LT-0265.

[19] X.L. Gou, W.T. Sun, Z. Chen, Y.G. Ju, Combust. Flame 157 (6) (2010) 1111-1121.

[20] W. Sun, Z. Chen, X.L. Gou, Y. Ju, Combust. Flame 157 (7) (2010) 1298-1307.

[21] W. Sun, Z. Chen, X.L. Gou, Y. Ju, The 6th US National Combustion Meeting, Ann Arbor, Michigan, USA, 2009; paper 22FA.

[22] S. Dooley, F. L. Dryer, T. I. Farouk, Y. Ju, S. H. Won, 51st AIAA Aerospace Science Meeting, 7-10 January 2013, Grapevine, Texas, AIAA 2013-0158.

[23] Z. Chen, M. P. Burke, Y.G. Ju, Combust. Inst. 32 (2009) 1253-1260.

[24] Z. Chen, "Studies on the Initiation, Propagation, and Extinction of Premixed Flames", PHD thesis, Princeton University, 2009.

[25] J. Kim and P. Moin, J. Comput. Phys. 59 (1985) 308-323. 
[26] M. P. Burke, Z. Chen, Y. Ju, and F. Dryer, Combust. Flame, 156 (2009) 771-779.

[27] Z. Chen, Combust. Flame, 158 (2011) 291-300.

[28] X.D. Liu, S. Osher and T. Chan, J. Comput. Phys, 115 (1994) 200-212.

[29] G.S. Jiang and C.W. Shu, J. Comput. Phys, 126 (1996) 202-228.

[30] D.S. Balsara and C.W. Shu, J. Comput. Phys, 160 (2000) 405-452.

[31] C.W. Shu, SIAM Review 51 (1) (2009) 82-126.

[32] C.W. Shu, "High Order ENO and WENO Schemes for Computational Fluid Dynamics", Springer, 1999.

[33] YA. B. Zeldovich, Combust. Flame 39 (1980) 211-214.

[34] J. Heywood, "Internal Combustion Engine Fundamentals", McGraw-Hill, 1988.

[35] F. M. Haas, A. Ramcharan, F. L. Dryer, Energy Fuels 25 (2011) 3909-3916.

[36] J. M. Burgers, "The Nonlinear Diffusion Equation, Asymptotic Solutions and Statistical Problems", Springer, 1974. 


\section{Figure 1}

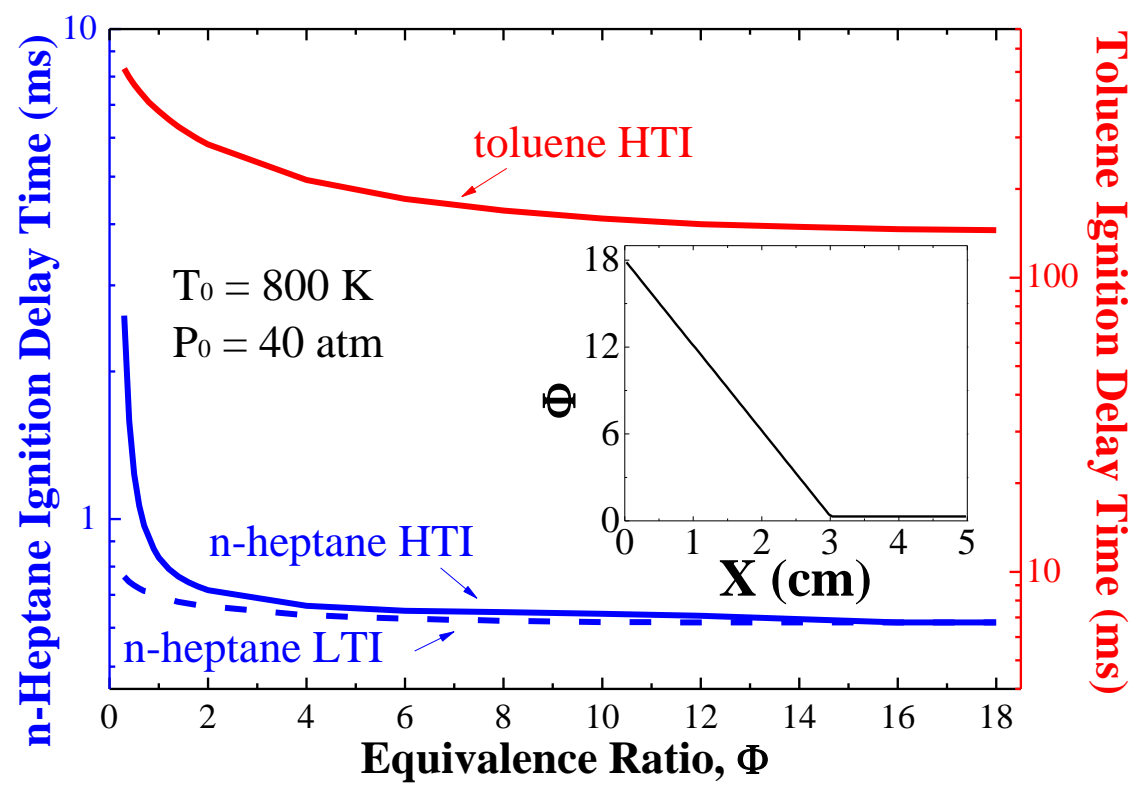




\section{Figure 2}

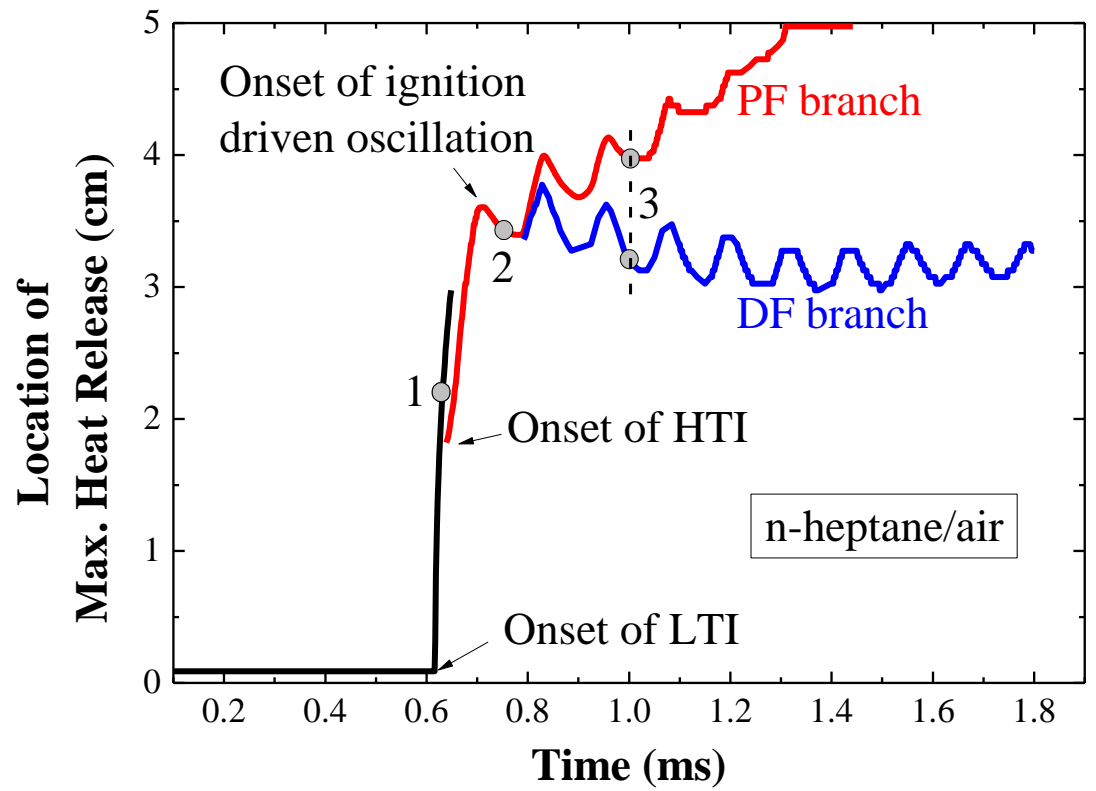




\section{Figure 3}
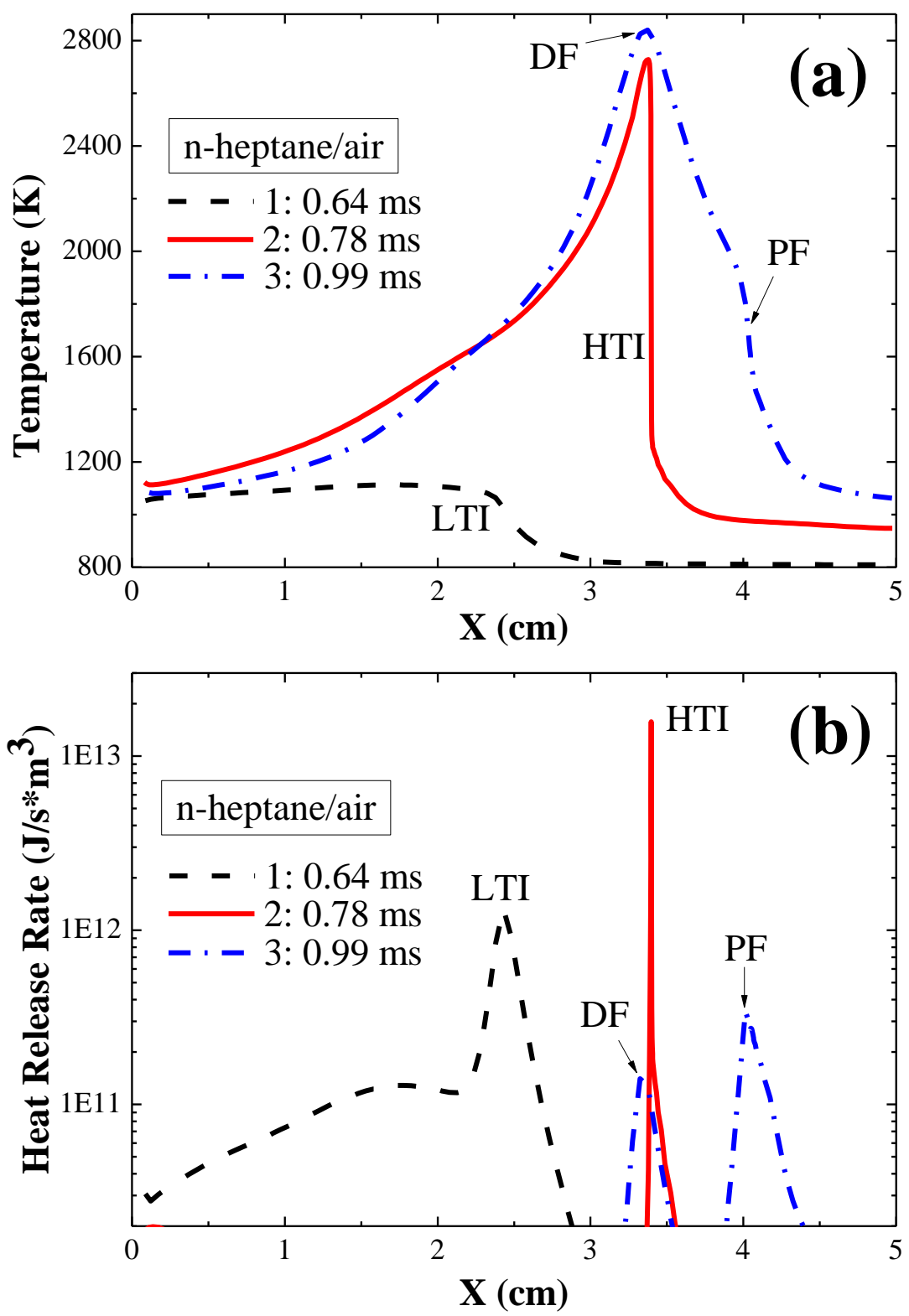


\section{Figure 4}
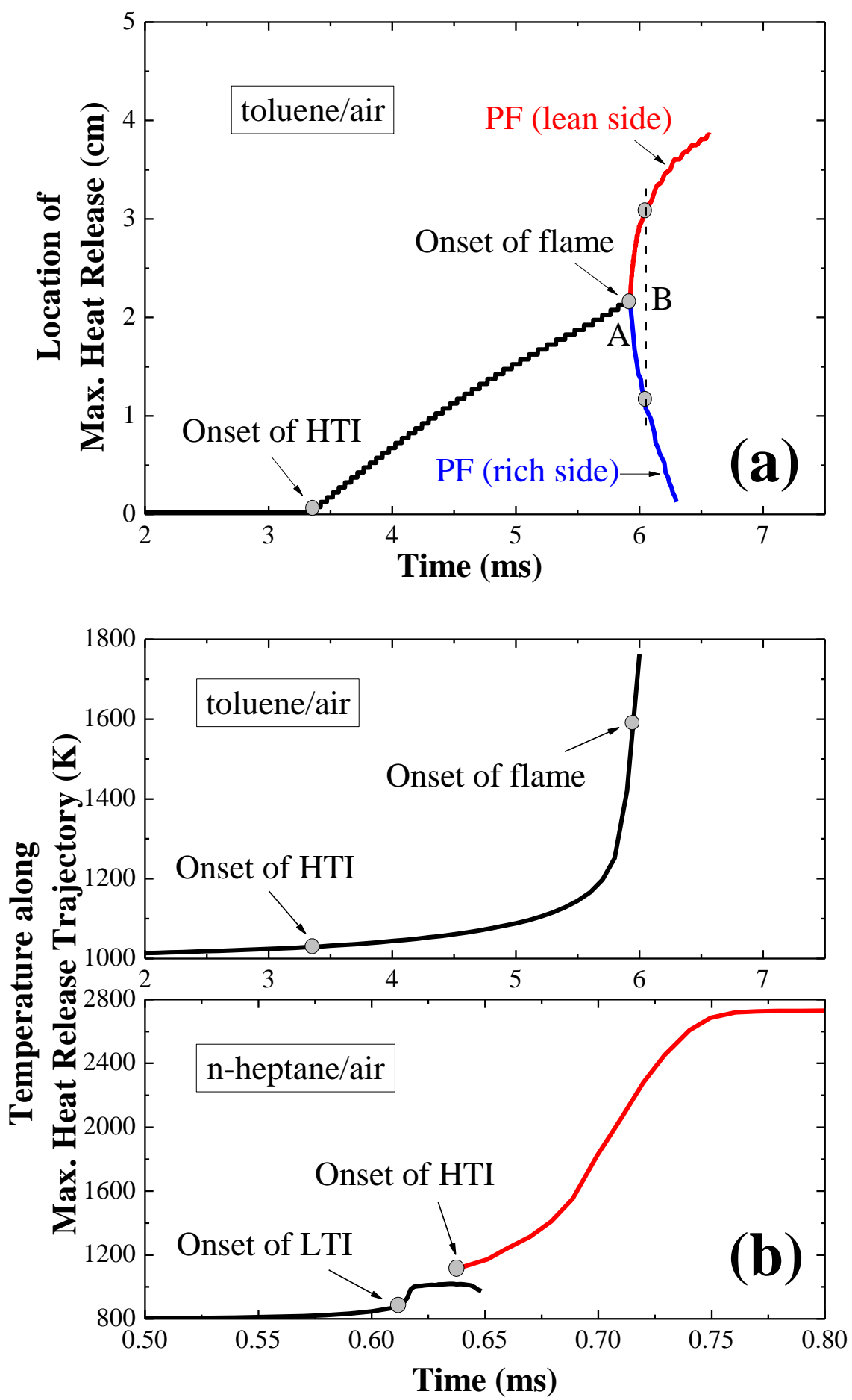


\section{Figure 5}
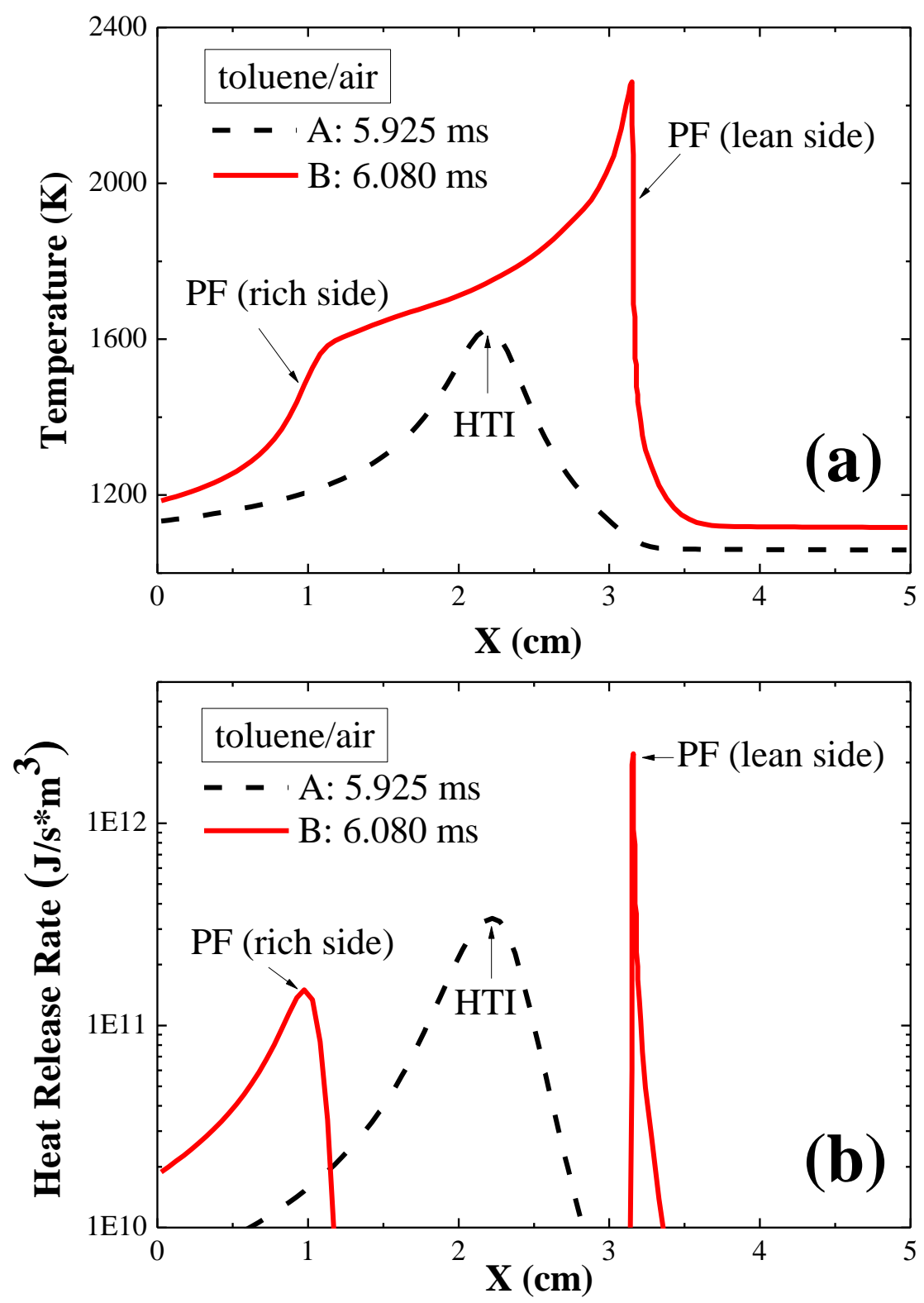


\section{Figure 6}
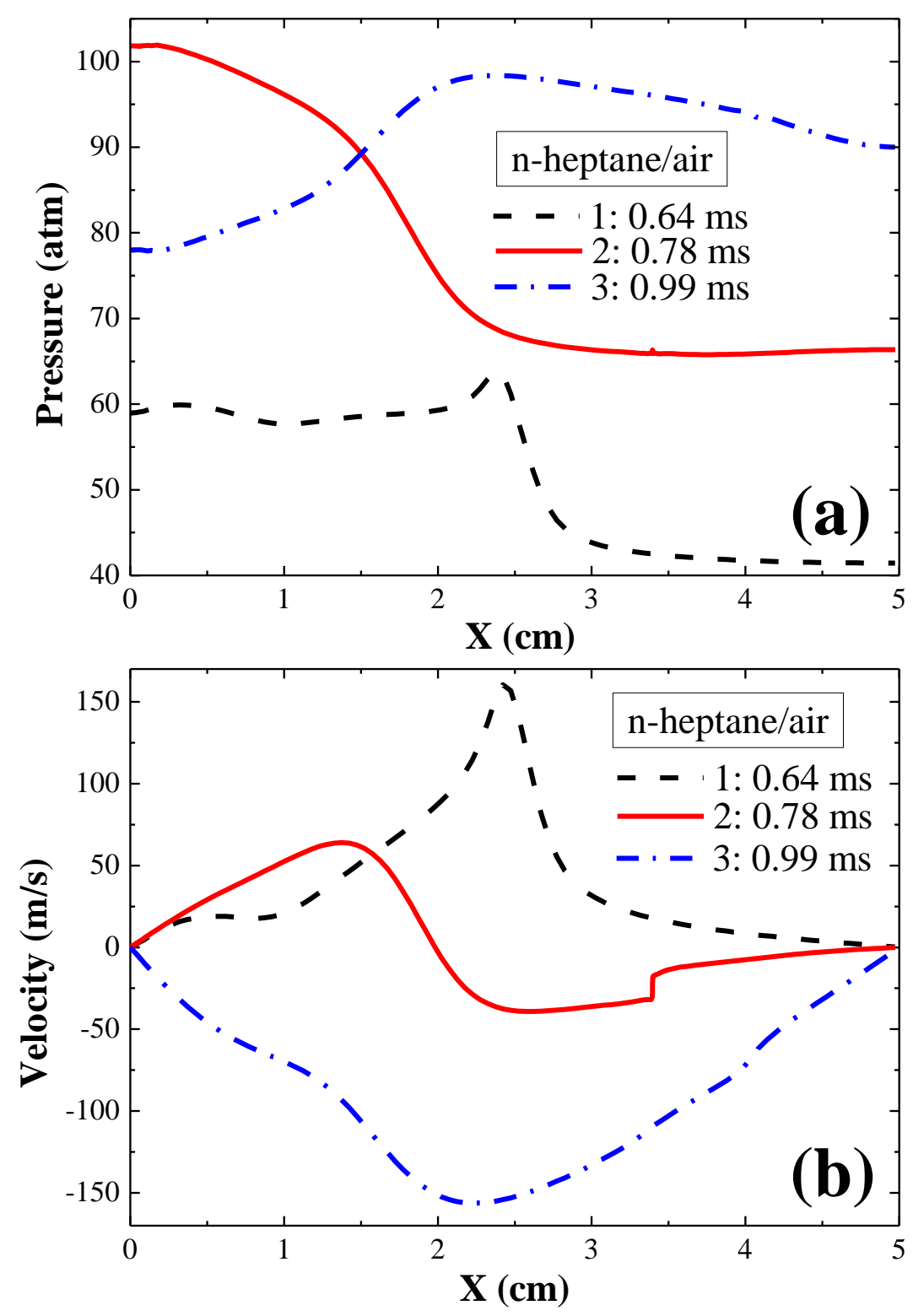


\section{Figure 7}

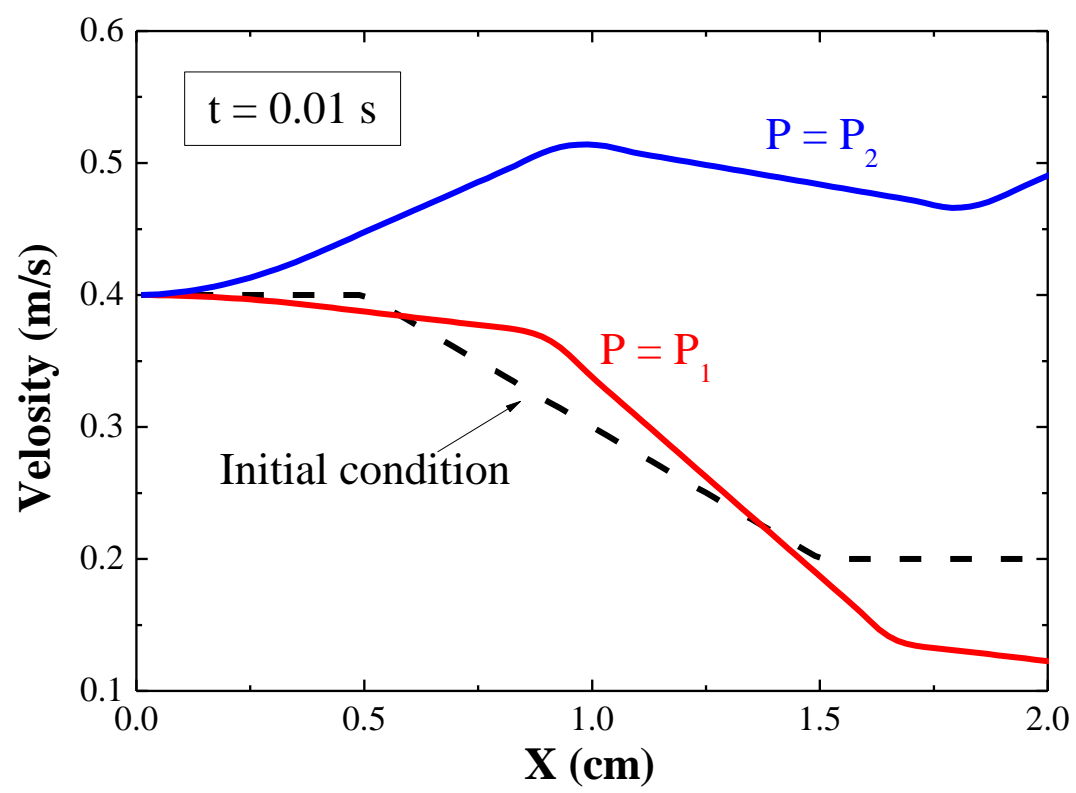

